\title{
Open challenges and good experimental practices in the research field of aqueous $\mathrm{Zn}$-ion batteries
}

\author{
Giorgia Zampardi (i] $^{1 凶} \&$ Fabio La Mantia (i) ${ }^{1,2 凶}$
}

Aqueous zinc-ion batteries are realistic candidates as stationary storage systems for power-grid applications. However, to accelerate their commercialization, some important challenges must be specifically tackled, and appropriate experimental practices need to be embraced to align the academic research efforts with the realistic industrial working conditions for stationary storage. Within this commentary article, both the open challenges and the good experimental practices are discussed in relation to their impact on the future development of the aqueous $\mathrm{Zn}$-ion technology.

In response to the increasing awareness regarding climate change, many countries have set the goal to increase the share of renewable energy within their total energy production ${ }^{1}$. Therefore, the development of cost-effective and safe energy storage technologies to accumulate the electrical energy harvested from renewable resources before putting it into the power grid has become a critical necessity of our society ${ }^{2,3}$.

In contrast to the already established Li-ion batteries, mild acidic aqueous $\mathrm{Zn}$-ion batteries (ZIBs) operating in a $\mathrm{pH}$ range of ca. $4-5.5^{4-6}$ are excellent candidates as storage systems for power grid applications. The reasons for this lay in their intrinsic high safety and environmentally friendliness, high specific power and high reversibility, non-toxicity, and most importantly, low costs and high abundancy of metallic zinc ${ }^{4,7,8}$. Indeed, the ZIB field has recently received great attention, as evidenced by the exponential growth of related publications in the last 10 years (Fig. 1).

The $\mathrm{Zn}$-ion concept usually consists of a $\mathrm{Zn}$-based negative electrode, onto/from which metallic zinc is electrodeposited and dissolved, and a positive electrode deinserting and inserting $\mathrm{Zn}^{2+}$ cations from/within its lattice during the battery cycling ${ }^{4,8,9}$. In mild acidic ZIBs, typical insertion materials for the positive electrodes are based on manganese oxide ${ }^{10}$, vanadium oxides $^{10}$, and Prussian blue analogs (PBAs) ${ }^{4}$.

Although the $\mathrm{Zn}$-ion concept can also be implemented with organic-based electrolytes, a realistic power-grid application of the $\mathrm{Zn}$-ion technology implies the use of water-based electrolyte solutions to ensure high safety levels and keep costs of the final devices at a minimum.

\footnotetext{
${ }^{1}$ Universität Bremen, Energiespeicher- und Energiewandlersysteme, Bibliothekstraße 1, 28359 Bremen, Germany. ${ }^{2}$ Fraunhofer Institute for Manufacturing

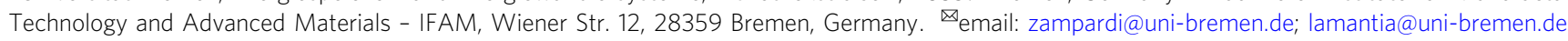




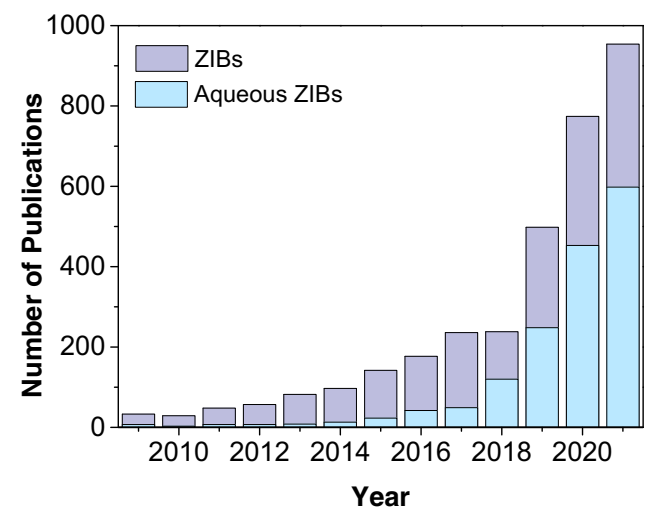

Fig. 1 Number of scientific peer-reviewed publications on ZIBs. Total number of scientific publications on ZIBs (organic- plus aqueous-based ZIBs) and specifically on aqueous ZIBs published in recent years (Data collected in November 2021 from Clarivate Web of Science. Keywords used: "zinc-ion batteries", "aqueous zinc-ion batteries").

In order to boost the commercialization of aqueous ZIBs as cheap and safe storage devices for the stationary grid, it is worth highlighting the challenges that remain yet to be addressed, together with the adoption of good experimental practices needed to align the academic research efforts with the industrial working conditions envisaged by a practical application of this battery technology.

\section{Current aqueous ZIBs limitations}

Specific energy and utilization of the zinc anode. Despite the clear advantages that aqueous ZIBs offer in terms of high safety and low costs, the current technology does not attain high enough specific energies (e.g., $>40 \mathrm{Wh} / \mathrm{kg}$ on full cell scale, including passive elements and casing) that are needed to access the stationary energy market.

The specific energy of a battery system is a function of the specific capacity of both the positive and the negative electrodes (often referred to as cathode and anode, respectively) together with the average discharge voltage of the cell. Although for a proper evaluation of the specific energy of the battery, the inactive components of the cell (such as contacts, current collector, case, etc.) must also be taken into account, Eq. 1 gives an acceptable first estimation of the battery specific energy:

$$
E=\frac{Q_{c a t} \times Q_{a n}}{Q_{c a t}+Q_{a n}} \times \Delta V_{\text {cell }}
$$

where: $Q_{c a t} Q_{a n}$ are the specific capacities of the active materials constituting the positive and the negative electrodes, respectively, and $\Delta \mathrm{V}_{\text {cell }}$ is the difference of the average operating potentials of the positive and the negative electrode, which defines the average discharge voltage of the cell.

In order to ensure low costs of the final aqueous ZIB, the use of a negative electrode based on metallic zinc is the obvious choice due to the high availability of metallic $\mathrm{Zn}$ and its low price (ca. $3.5 \$ / \mathrm{kg}^{11}$ ).

Current academic research efforts to increase the energy content of aqueous $\mathrm{Zn}$-ion batteries generally focus on developing new cathode materials with high specific capacity (e.g., $>200 \mathrm{Ah} /$ $\left.\mathrm{kg}^{9}\right)$. At the same time, however, the role of the utilization of the zinc-based negative electrode and of its Coulombic efficiency on the electrochemical performance of the battery is widely ignored, despite its heavy influence on the specific energy of a full $\mathrm{Zn}$-ion cell (Eq. 1). The Zn-based anodes routinely employed consist of $\mathrm{Zn}$ foils, metallic zinc deposited on a substrate, or composite electrodes with $\mathrm{Zn}$ particles within their formulation ${ }^{7,9}$. In order to understand the great challenges that hide behind the $\mathrm{Zn}$-based anode utilization (often referred to as depth of discharge, DOD), it is helpful to consider the equi-energy curves, which are a graphical representation of Eq. 1. Such curves can be calculated as a function of the specific capacity of a generic cathode, as shown in Fig. 2. In each graph, a different utilization (or depth of discharge) of the zinc anode has been taken into consideration. Figure 2a represents the ideal scenario, in which the zinc anode is fully used (100\% utilization or depth of discharge) and perfectly matched in terms of charge with the cathode. Figure $2 b$ is the envisaged practical scenario in which the anode is utilized at $50 \%$, meaning that it is two times oversized with respect to the cathode. This means that the total mass of the active material within the electrode formulation (metallic $\mathrm{Zn}$ in the case of the anode) is two times more than the one needed to balance the capacity of the cathode. Figure $2 \mathrm{c}$ and Fig. $2 \mathrm{~d}$ represent the more common, worse scenarios in which only 25 and $12.5 \%$ of the anode is used, corresponding to four times and eight times oversized anode with respect to the cathode, respectively.

Equation 1 is also helpful when new cathode materials are developed. As it can be seen in its graphical representation in Fig. 2, developing high specific capacity cathode materials $\left(Q_{\text {cat }}>100 \mathrm{Ah} / \mathrm{kg}\right)$ has no practical significance if their operating potential is not high enough to lead to an average discharge voltage of the $\mathrm{Zn}$-ion cell at least higher than 1.0-1.2 V. To make an example, a cathode material having $300 \mathrm{Ah} / \mathrm{kg}$ with a working potential of $0.6 \mathrm{~V}$ vs $\mathrm{Zn} / \mathrm{Zn}^{2+}$ tested with a $\mathrm{Zn}$ anode utilized at $50 \%$ (practical envisaged scenario) would lead to a $\mathrm{Zn}$-ion cell with specific energy lower than $100 \mathrm{Wh} / \mathrm{kg}$ (Fig. 2b). Higher specific energy could be reached with a cathode material having only $100 \mathrm{Ah} / \mathrm{kg}$ but with a working potential of $1.5 \mathrm{~V}$ vs $\mathrm{Zn} / \mathrm{Zn}^{2+}$, keeping the same utilization of the zinc anode (50\%). This effect is evident in the case of the lower zinc anode utilization (Fig. 2d), as the performance of the $\mathrm{ZIB}$ is dominated by the $\mathrm{Zn}$ anode itself.

Unfortunately, the operational conditions that are mostly used in academic research employ an anode even more than eight times oversized with respect to the cathode, as the positive electrode is usually tested with low active mass loadings of around $1-2 \mathrm{mg} / \mathrm{cm}^{2}$ while a metallic $\mathrm{Zn}$ foil anode usually has a $\mathrm{Zn}$ loading up to $1000 \mathrm{mg} / \mathrm{cm}^{29}$.

Parasitic hydrogen evolution and irreversible $\mathrm{Zn}$-ion losses. Another important challenge that is often ignored within the aqueous ZIB literature concerns the main parasitic reaction occurring within a $\mathrm{Zn}$-ion cell, namely: hydrogen evolution occurring at the zinc-based anode.

The unwanted, although thermodynamically favored $\mathrm{H}_{2}$ evolution reaction occurs mainly during the zinc deposition step. Due to the reduction of the hydronium ion $\mathrm{H}^{+}$(aq.) to gaseous $\mathrm{H}_{2}$, the $\mathrm{pH}$ in the proximity of the $\mathrm{Zn}$-based anode locally increases up to values of ca. $6-7.5^{12}$. In this $\mathrm{pH}$ range, the formation and the stabilization of inactive and/or poorly conductive zinc passivation species and clay-like layered double hydroxides are strongly favored ${ }^{13-17}$. This not only causes an irreversible loss of $\mathrm{Zn}^{2+}$ from the electrolyte, but also a decrease of the Coulombic efficiency of the $\mathrm{Zn}$ anode and thus of the whole $\mathrm{Zn}$-ion cell.

It is worth mentioning that due to the local increase of the $\mathrm{pH}$ caused by the occurrence of the $\mathrm{H}_{2}$ evolution reaction, the evolution of the morphology of the $\mathrm{Zn}$-based anode during the cycles often shows the appearance of non-homogeneous lamellar deposits, which are typical of the poorly conductive/inactive layered double hydroxides ${ }^{13-15}$, and less commonly of fine dendrites $^{18}$. 

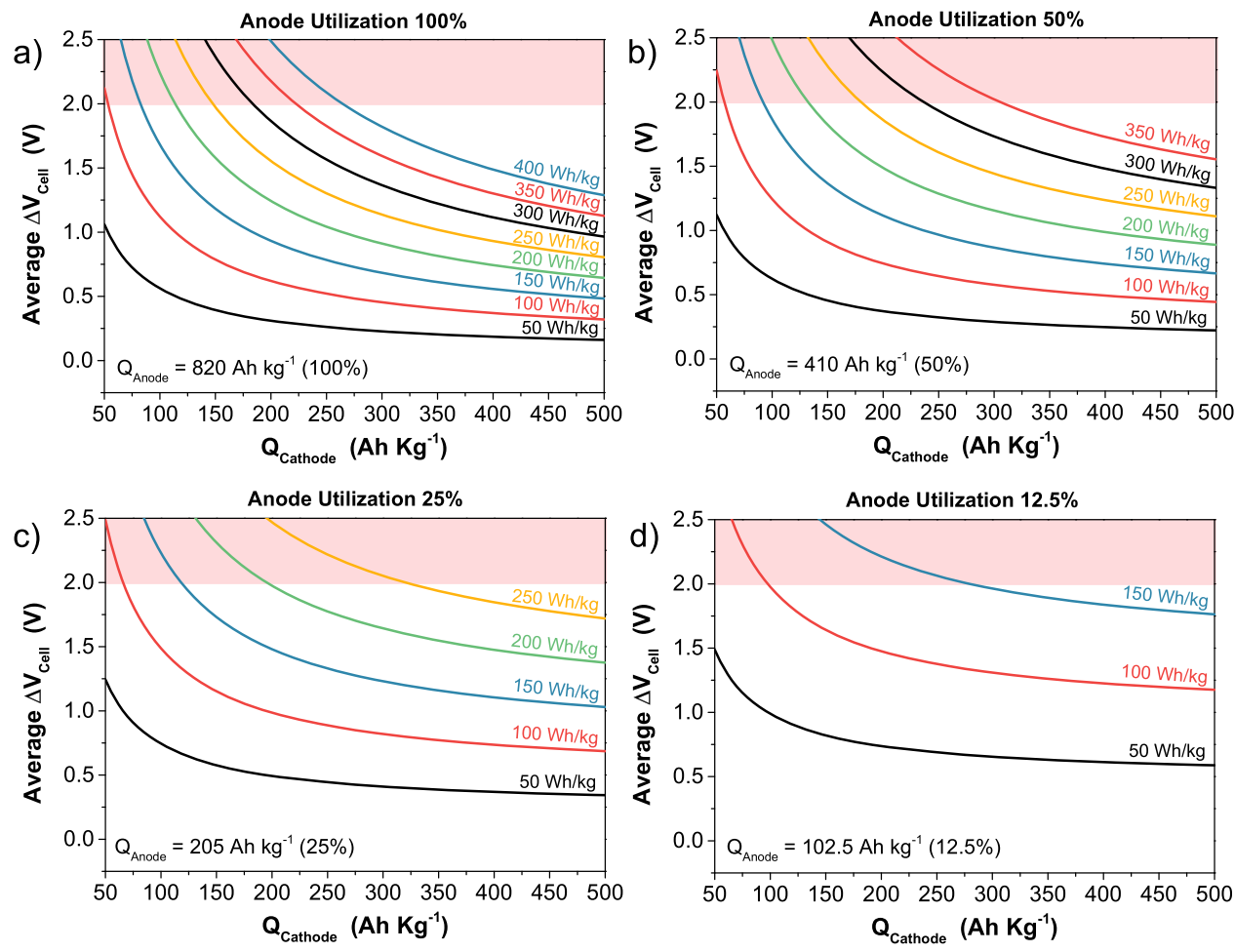

Fig. 2 Equi-energy curves of ZIBs. Equi-energy curves of a ZIB calculated from Eq. 1 as a function of the specific charge capacity of a generic cathode. Each graph represents a different extent of utilization of a metallic zinc anode, namely: a $100 \%, \mathbf{b} 50 \%$, c $25 \%$, and $\mathbf{d} 12.5 \%$. Shaded in red is the region in which water splitting occurs, an inaccessible operating regime for ZIBs.

\section{Good experimental practices}

Realistic current rates (C-rates) during the electrochemical tests. The current rate (C-rate) represents the inverse of the time in hours needed by the battery to complete a full discharge or a full charge. It is very important to perform power rate experiments in order to evaluate the efficiency and capacity losses of the electrodes when subjected to different cycling rates, as this represents a realistic working scenario for a battery intended for power grid applications.

However, it is of no practical interest to perform cycle-life duration experiments at C-rates higher than $10 \mathrm{C}$ (meaning full charge or discharge of the battery occurring in $6 \mathrm{~min}$ ), as the operational currents required by a storage device for the power grid are around $0.5-2 \mathrm{C}$ and do not exceed $10 \mathrm{C}^{19}$. Long cycle life ( $\geq 1000$ cycles) of the cathode materials is very often claimed in the literature based on C-rates much higher than $10 \mathrm{C}$, up to ca. $100 \mathrm{C}$ (full charge or discharge of the electrodes in $36 \mathrm{~s}$ ), where capacitive effects of the double layer often mask the ones related to the Faradic reactions. Moreover, the high current rates strongly underestimate the chemical degradation of the materials, simply because degradation processes depend on time and not on the number of cycles.

However, it is generally known that the higher the C-rate, the slower the aging processes of an insertion material ${ }^{9,20,21}$. In order to collect non-misleading and meaningful results on a realistic estimation of the cycle life of anode and cathode materials, it is critical to choose a $1 \mathrm{C}$ rate for the aging test, as this mimics the rate required for aqueous ZIBs intended to be used as storage devices for the power grid.

Mass loading of the active materials. The relative mass loading of the active materials for both the anode and the cathode is a very important aspect, which strongly affects both the cycle life of the electrodes and the specific energy of the $\mathrm{Zn}$-ion cell. Despite its clear importance on the cell's performance, the active mass loading of the electrodes is not generally given proper consideration in the literature.

The electrodes employed for lab-scale measurements often contain a low active material mass loading of ca. $1 \mathrm{mg} / \mathrm{cm}^{2}$. When such electrodes are electrochemically tested they experience negligible diffusion limitations and increased electrical connectivity among the different particles, which results in negligible polarization effects. This behavior artificially improves the performance of the material under investigation ${ }^{9,20}$. A realistic evaluation of ZIBs, and in particular of aqueous ZIBs, requires electrodes with an active material mass loading of at least ca. $7-10 \mathrm{mg} / \mathrm{cm}^{29,20}$. The mass loading should be high enough that an areal capacity of $2-4 \mathrm{mAh} / \mathrm{cm}^{2}$ is reached.

Electrodes balancing and amount of $\mathrm{Zn}$ ions involved during cycling. As already pointed out in the previous section, the relative mass loading of both anode and cathode should be balanced in terms of charge.

As clearly demonstrated by Eq. 1 and Fig. 2, the smaller the mass of the active material within the cathode with respect to the active mass of the anode, the smaller the utilization (depth of discharge) of the anode. Consequently, the more unbalanced the electrodes, the lower the specific energy of the Zn-ion cell. Unfortunately, it is a very common practice to work with cathodes having active material loadings of around hundred-folds smaller than the active mass of the anode (e.g., cathode active material around $1-2 \mathrm{mg} / \mathrm{cm}^{2}$, coupled with an anode active mass of ca. $150-300 \mathrm{mg} / \mathrm{cm}^{2}$ ). Unbalanced electrodes are unrealistic as they not only yield artificially high electrochemical performance and Coulombic efficiency of both electrodes (though this effect is more drastic for the Zinc electrodeposition/dissolution process at the anode) but also drastically reduce the specific energy of the $\mathrm{Zn}$-ion cell and vastly increase its costs. 
Reproducibility of the electrochemical tests and data representation. In order to demonstrate the reproducibility of the electrochemical results disclosed in a scientific report or publication, the defining cycling performance characteristics of a material (such as specific capacity, capacity retention, Coulombic efficiency, etc.) should be always reported with mean values and standard deviations averaged over at least three different experiments ${ }^{21}$. In this way, the reproducibility of the acquired measurements would be clearly demonstrated and not only claimed.

Moreover, quantities such as the Coulombic efficiency should be correctly displayed with an appropriate resolution, implying the use of a scale with appropriate dimensions that do not span from 0 to $>120 \%$. Only in this way, the data are represented clearly enough to distinguish the possible fluctuation of efficiencies that could be related to important physical phenomena occurring at the electrodes (such as passivation of the surface of the electrode, occurrence of parasitic reactions, degradation of additives in the electrode/electrolyte, etc.).

Last but not the least, the electrochemical analysis of $\mathrm{Zn}$-ion batteries electrodes should be carried out not only in flooded cells containing excess electrolyte (usually around $10 \mathrm{ml}$, corresponding to ca. $5 \mathrm{ml} / \mathrm{cm}^{2}$ with respect to the geometric area of the electrodes), but also in compact ones, where "electrolyte starving" conditions are attained (usually around $50-100 \mu \mathrm{l} / \mathrm{cm}^{2}$ with respect to the geometric area of the electrodes) ${ }^{9}$. During the experiments carried out in cells with electrolyte starving conditions the detrimental effect of the parasitic reactions, leading to the loss of active $\mathrm{Zn}^{2+}$ due to the formation of zinc oxide/hydroxide-based inactive compounds, is amplified due to the smaller amount of $\mathrm{Zn}^{2+}$-containing electrolyte. This would then result in lower cycle life and lower Coulombic efficiencies with respect to the case where electrolyte flooded conditions are used for the ZIB electrodes analysis?.

\section{Summary and outlook}

Due to their promising characteristics, mild aqueous ZIBs represent a viable, green, and cost-effective energy storage technology for stationary grid applications. In order to quickly and efficiently reach this goal, it is worth focusing on the remaining challenges: the increase of the specific energy of the full $\mathrm{Zn}$-ion cell, and the prevention of the parasitic $\mathrm{H}_{2}$ evolution reaction occurring during the $\mathrm{Zn}$ electrodeposition step.

In order to do so, particular attention must be taken in cycling a fair amount of $\mathrm{Zn}^{2+}$ contained within the $\mathrm{Zn}$-based anode with respect to its total mass and in developing cathode materials resulting in an average discharge voltage of the cell at least $\geq 1.0-1.2 \mathrm{~V}$. Moreover, research efforts should also address the optimization of the electrolyte composition (e.g., additives), towards the development of future water-based electrolytes with the aim to hinder the parasitic hydrogen evolution reaction at the anode.

In order to produce meaningful experimental results, which represent realistic ZIB working conditions, the electrochemical tests should be standardized. In particular: (i) the C-rates employed for the cycle-life assessment of the materials should reflect the need for a stationary power grid (i.e., long-term cycling tests at around 1C must be carried out); (ii) the electrodes employed during the electrochemical tests should have a realistic amount of active material mass loading (e.g., in the $7-15 \mathrm{mg} / \mathrm{cm}^{2}$ range); (iii) both electrodes (anode and cathode) of the electrochemical cell should be balanced in terms of charge. Moreover, attention should be paid to testing $\mathrm{Zn}$-ion battery electrodes and full cells not only in flooded conditions but also in cells where "electrolyte starving" conditions are attained, as this would mimic the real working conditions of the battery.
On the other side, in order to demonstrate the robustness and reproducibility of the research findings, experimental data (such as Coulombic efficiency, specific capacity, specific capacity retention, etc.) should always be averaged over at least three different measurements.

Only through focusing on the main issues that are currently limiting the practical application of aqueous ZIBs and the adoption of good experimental practice in the academic research, which reflects the real working conditions envisaged for stationary power grid applications, researchers will be able to push forward the development of the aqueous $\mathrm{Zn}$-ion technology, allowing it to permeate the stationary energy market.

Received: 2 November 2021; Accepted: 21 January 2022; Published online: 03 February 2022

\section{References}

1. Eurostat. Infographs. https://ec.europa.eu/eurostat/cache/infographs/energy/ bloc-4c.html (2021).

2. BATTERY 2030+: a long-term roadmap for forward-looking battery research in Europe. https://battery2030.eu/research/roadmap/ (2021).

3. Blay, V. et al. Research frontiers in energy-related materials and applications for 2020-2030. Adv. Sustain. Syst. 4, 1900145 (2020).

4. Zampardi, G. \& La Mantia, F. Prussian blue analogues as aqueous Zn-ion batteries electrodes: current challenges and future perspectives. Curr. Opin. Electrochem. 21, 84-92 (2020).

5. Kasiri, G., Glenneberg, J., Kun, R., Zampardi, G. \& La Mantia, F. Microstructural changes of Prussian blue derivatives during cycling in zinccontaining electrolytes. ChemElectroChem 7, 3301-3310 (2020).

6. Lim, J. et al. Irreversible structural changes of copper hexacyanoferrate used as a cathode in Zn-Ion batteries. Chemistry 26, 4917-4922 (2020).

7. Zampardi, G. \& Compton, R. G. Fast electrodeposition of zinc onto single zinc nanoparticles. J. Solid State Electrochem. 24, 2695-2702 (2020).

8. Shin, J., Lee, J., Park, Y. \& Choi, J. W. Aqueous zinc ion batteries: focus on zinc metal anodes. Chem. Sci. 11, 2028-2044 (2020).

9. Li, Y. et al. Understanding the gap between academic research and industrial requirements in rechargeable zinc-ion batteries. Batteries Supercaps 4, 60-71 (2020).

10. Ming, J., Guo, J., Xia, C., Wang, W. \& Alshareef, H. N. Zinc-ion batteries: materials, mechanisms, and applications. Mater. Sci. Eng. R Rep. 135, 58-84 (2019).

11. Metalle IfsEus. Price for base metals in December 2020. https://en.institutseltene-erden.de/prices-for-base-metals-in-december/ (2021).

12. Argoul, F. \& Kuhn, A. Experimental demonstration of the origin of interfacial rhythmicity in electrodeposition of zinc dendrites. J. Electroanal. Chem. 359, 81-96 (1993).

13. Hashemi, A. B. et al. Electrochemical and morphological characterization of $\mathrm{Zn}-\mathrm{Al}-\mathrm{Cu}$ layered double hydroxides as a negative electrode in aqueous zincion batteries. ChemElectroChem 5, 2073-2079 (2018).

14. Hashemi, A. B., Kasiri, G. \& La Mantia, F. The effect of polyethyleneimine as an electrolyte additive on zinc electrodeposition mechanism in aqueous zincion batteries. Electrochim. Acta 258, 703-708 (2017).

15. González, M. A., Trócoli, R., Pavlovic, I., Barriga, C. \& La Mantia, F. Layered double hydroxides as a suitable substrate to improve the efficiency of $\mathrm{Zn}$ anode in neutral pH Zn-ion batteries. Electrochem. Commun. 68, 1-4 (2016).

16. Oberholzer, P., Tervoort, E., Bouzid, A., Pasquarello, A. \& Kundu, D. Oxide versus nonoxide cathode materials for aqueous $\mathrm{Zn}$ batteries: an insight into the charge storage mechanism and consequences thereof. ACS Appl Mater. Interfaces 11, 674-682 (2019).

17. Moezzi, A., Cortie, M. B. \& McDonagh, A. M. Zinc hydroxide sulphate and its transformation to crystalline zinc oxide. Dalton Trans. 42, 14432-14437 (2013).

18. Yang, Q. et al. Do zinc dendrites exist in neutral zinc batteries: a developed electrohealing strategy to in situ rescue in-service batteries. Adv. Mater. 31, e1903778 (2019).

19. Hesse, H., Schimpe, M., Kucevic, D. \& Jossen, A. Lithium-ion battery storage for the grid-A review of stationary battery storage system design tailored for applications in modern power grids. Energies 10, 2107 (2017).

20. Lin, Z., Liu, T., Ai, X. \& Liang, C. Aligning academia and industry for unified battery performance metrics. Nat. Commun. 9, 5262 (2018).

21. Zampardi, G. et al. Effect of the reactants concentration on the synthesis and cycle life of copper hexacyanoferrate for aqueous $\mathrm{Zn}$-ion batteries. Electrochem. Commun. 126, 107030 (2021). 


\section{Acknowledgements}

The financial support of the Federal Ministry of Education and Research (BMBF) in the framework of the project "ZIB" (FKZ 03XP0204A) is gratefully acknowledged.

\section{Author contributions}

G.Z. and F.L.M. contributed equally in the discussion of the data and in the writing and the conceptualization of this article.

\section{Competing interests}

The authors declare no competing interests.

\section{Additional information}

Correspondence and requests for materials should be addressed to Giorgia Zampardi or Fabio La Mantia.

Reprints and permission information is available at http://www.nature.com/reprints

Publisher's note Springer Nature remains neutral with regard to jurisdictional claims in published maps and institutional affiliations.

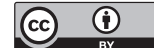

(c) distribution and reproduction in any medium or format, as long as you give appropriate credit to the original author(s) and the source, provide a link to the Creative Commons license, and indicate if changes were made. The images or other third party material in this article are included in the article's Creative Commons license, unless indicated otherwise in a credit line to the material. If material is not included in the article's Creative Commons license and your intended use is not permitted by statutory regulation or exceeds the permitted use, you will need to obtain permission directly from the copyright holder. To view a copy of this license, visit http://creativecommons.org/ licenses/by/4.0/.

(C) The Author(s) 2022 